\title{
Nuclear Imaging and Minimally Invasive Surgery in the Management of Hyperparathyroidism*
}

\author{
Benjamin L. Judson and Ashok R. Shaha \\ Head and Neck Service, Memorial Sloan-Kettering Cancer Center, New York, New York
}

Primary hyperparathyroidism is the most common cause of hypercalcemia, and the treatment is primarily surgical. Because of biochemical screening, more patients now present with asymptomatic primary hyperparathyroidism, and consensus guidelines have been developed for the treatment of these patients. There is now considerable interest in minimally invasive approaches to the treatment of hyperparathyroidism. Sestamibi scanning as a localizing study, used in combination with anatomic imaging and intraoperative rapid parathyroid hormone assays, has enabled focused surgical approaches. Patients with localizing studies that indicate a single parathyroid adenoma are candidates for such approaches, including unilateral neck exploration, minimally invasive single-gland exploration, or endoscopic exploration instead of the traditional approach of bilateral neck exploration. Nuclear imaging is also critical to the successful management of patients with persistent or recurrent hyperparathyroidism.

Key Words: endocrinology; hyperparathyroidism; sestamibi; endoscopic exploration; minimally invasive single-gland exploration; parathyroid hormone assays

J Nucl Med 2008; 49:1813-1818

DOI: 10.2967/jnumed.107.050237

$\mathbf{P}$ rimary hyperparathyroidism is characterized by the autonomous production of parathyroid hormone resulting in hypercalcemia. This is a common disease, occurring in about $1 \%$ of the adult population and about $2 \%$ of the population older than $55 \mathrm{y}(1)$. It occurs $2-3$ times more frequently in women than in men. Hyperparathyroidism peaks in incidence in the fourth and fifth decades of life but can occur in young children and the elderly as well (2). Historically, patients presented with symptoms such as

\footnotetext{
Received May 13, 2008; revision accepted Sep. 2, 2008.

For correspondence or reprints contact: Ashok R. Shaha, FACS Head and Neck Service, Memorial Sloan-Kettering Cancer Center, 1275 York Ave., New York, NY.

E-mail: shahaa@mskcc.org

*NOTE: FOR CE CREDIT, YOU CAN ACCESS THIS ACTIVITY THROUGH THE SNM WEB SITE (http://www.snm.org/ce_online) THROUGH NOVEMBER 2009.

The authors have indicated no relevant relationships that could be perceived as a real or apparent conflict of interest. No other potential conflict of interest relevant to this article was reported.

COPYRIGHT @ 2008 by the Society of Nuclear Medicine, Inc.
}

urolithiasis, bone pain, and pathologic fractures and nonspecific symptoms such as depression, lethargy, and vague aches and pains. Since the advent of multichannel biochemical screening, however, patients frequently present without symptoms after being found to have hypercalcemia on routine laboratory screening (3). The frequency of this previously uncommon clinical scenario, an asymptomatic patient with hyperparathyroidism, has led to controversy and changes in the management of patients with this disease. These changes will be reviewed and further discussed in the "Treatment" section of this article.

In addition to causing these changes in the clinical presentation of primary hyperparathyroidism, technology has also led to changes in the treatments used for this disease. Surgery has been, and continues as, the most commonly used and successful treatment. The use of nuclear imaging, in combination with improved ultrasound imaging and the availability of rapid intraoperative parathyroid hormone assays, has changed the strategy of surgical treatment of primary hyperparathyroidism (3-5). Previously, the standard treatment for all patients with primary hyperparathyroidism was a bilateral neck exploration with the goal of identifying and evaluating 4 parathyroid glands. Visual inspection of the glands, sometimes used in conjunction with intraoperative histologic assessment of frozen sections, allowed experienced surgeons to identify the pathologic glands and remove them with a success rate in excess of $90 \%$ (3). The ability to preoperatively localize pathologic parathyroid glands has enabled a more focused surgical approach. Most centers now use preoperative imaging and intraoperative rapid testing of parathyroid hormone with either a unilateral neck exploration or, increasingly, a minimally invasive single-gland exploration for the treatment of patients with primary hyperparathyroidism. Endoscopic parathyroid exploration is performed at a few centers but has not been widely adopted. These focused surgical approaches are possible only when preoperative nuclear imaging suggests the presence of a localized parathyroid adenoma.

\section{ANATOMY}

Successful localization and surgical management requires an understanding of the anatomy and development of the parathyroid glands. The parathyroid glands develop 
during the sixth week of gestation. The superior glands develop from the fourth brachial pouch, along with the ultimobrachial bodies, which become the $\mathrm{C}$ cells in the thyroid gland. The inferior parathyroid glands develop from the third brachial pouch, along with the thymus. Because of this relationship, the superior parathyroid gland remains near the superior pole of the thyroid lobe. Occasionally, the superior gland may remain undescended near the hyoid bone, along the pharyngeal musculature described as a parapharyngeus, or anywhere along its route of descent adjacent to the carotid sheath (Table 1). Less commonly, the superior gland can descend inferior to the inferior gland. The inferior parathyroid glands have a more varied anatomy and are more commonly found in ectopic sites. The most common ectopic location of the inferior parathyroid gland is within the thymic capsule or the superior mediastinum. Even though there are typically 4 parathyroid glands, approximately $10 \%$ of individuals have between 5 and 7 glands, known as supernumerary parathyroid glands, and $2 \%-3 \%$ of individuals have fewer than 4 glands (6).

\section{PATHOPHYSIOLOGY}

The parathyroid glands secrete parathyroid hormone (PTH), which tightly regulates calcium levels in the body. PTH acts on bones, in which it stimulates bone remodeling and the release of calcium. PTH also acts on the kidneys, in which it stimulates calcium resorption and decreases resorption of phosphate. PTH stimulates the synthesis of the active form of vitamin $\mathrm{D}$, called 1,25-dihydroxyvitamin $\mathrm{D}$, which in turn stimulates calcium absorption in the gastrointestinal tract.

Primary hyperparathyroidism occurs with autonomous hypersecretion of PTH from 1 or more parathyroid glands. Hyperparathyroidism is classified as secondary hyperparathyroidism when the parathyroid glands hypersecrete PTH in response to a nonparathyroid gland pathology, which causes hypocalcemia, such as chronic renal failure. Tertiary hyperparathyroidism is the autonomous production of PTH after a period of secondary hyperparathyroidism. An example of tertiary disease is patients who have received a kidney transplant after a long period of secondary hyperparathyroidism due to renal failure. The prolonged hypersecretion in response to renal failure can lead to continued autonomous secretion even after the renal failure is corrected (7).

\section{TABLE 1}

Location of Parathyroid Glands

\begin{tabular}{ll}
\hline \multicolumn{1}{c}{ Superior parathyroid gland } & Inferior parathyroid gland \\
\hline Adjacent to superior thyroid pole & Retromanubrial space \\
Tracheoesophageal groove & Thymic fat pad \\
Retroesophageal & Mediastinum \\
Carotid sheath & Within thyroid gland \\
Within thyroid gland & \\
\hline Adapted from Shaha (6). & \\
\hline
\end{tabular}

Approximately $85 \%$ of patients with primary hyperparathyroidism have a single parathyroid gland adenoma. Approximately $6 \%$ of patients have 4 -gland hyperplasia, and $4 \%$ have double adenomas. Less than $1 \%$ of patients presenting with primary hyperparathyroidism have parathyroid carcinoma. It is frequently difficult to distinguish histologically between hyperplastic parathyroid gland, adenoma, or carcinoma. All have an increased density of parathyroid cells replacing the fat encountered in healthy glands. However, the effective treatment of any of these diseases requires identification of all the pathologically involved glands.

\section{DIAGNOSIS}

Primary hyperparathyroidism is the most common cause of hypercalcemia in the ambulatory setting. Nonetheless, the differential diagnosis of hypercalcemia is complex and includes conditions such as metastatic cancer, multiple myeloma, sarcoidosis and other granulomatous diseases, ingestion of calcium or vitamin D, milk-alkali syndrome, and other less common causes. In contradistinction to almost all other causes, however, patients with primary hyperparathyroidism present with both elevated calcium and elevated or inappropriately normal parathyroid hormone level (7). When the parathyroid glands are functioning normally, high serum calcium levels will suppress PTH levels. Therefore, if both PTH and calcium levels are elevated, this indicates hyperparathyroidism. Patients with primary hyperparathyroidism also typically have low phosphorus, because elevated PTH levels decrease the resorption of phosphorus in the kidneys.

Parathyroid hormone levels are measured using an immunoradiometric assay that recognizes either the entire PTH protein or a large fragment of the protein truncated at the N-terminal. Assays that measure only intact PTH protein are the preferred method for measuring PTH levels. Although there is some debate as to whether it is superior to measure ionized calcium or to measure total serum calcium and correct for the patient's albumin concentration, the latter approach is still frequently used. The correction is made by adding $0.8 \mathrm{mg} / \mathrm{dL}$ to the total serum calcium value for every $1 \mathrm{~g} / \mathrm{dL}$ below a serum albumin concentration of $4 \mathrm{~g} / \mathrm{dL}$. Aside from primary hyperparathyroidism, there are a few other uncommon disorders that can present with an elevation of both calcium and PTH levels. One such disorder is familial hypocalciuric hypercalcemia. With this condition, patients will have abnormally elevated levels of both calcium and PTH. However, analysis of 24-h urine calcium will reveal abnormally low levels of calcium in the urine. Because surgery is not indicated in patients with familial hypocalciuric hypercalcemia, it is important to evaluate patients suspected of having primary hyperparathyroidism by $24-\mathrm{h}$ urine calcium analysis. Patients on lithium or thiazide diuretics can also present with elevated calcium and PTH. If it is considered medically safe, these patients can stop the medications to determine whether they have hyperparathyroidism. Elevated calcium and PTH after the patient has discontinued the thiazide or lithium indi- 
cates the presence of an underlying primary hyperparathyroidism. Patients with tertiary hyperparathyroidism, such as when a patient has a history of secondary hyperparathyroidism due to renal failure and subsequently undergoes a kidney transplant, will also have elevated calcium and PTH (2). Paraneoplastic hypercalcemia resulting from the production of parathyroid hormone-related protein by tumor cells is generally not a diagnostic consideration, because parathyroid hormone-related protein is not detected by the clinical assays used for measuring PTH.

\section{LOCALIZING STUDIES}

Sestamibi, since its introduction for parathyroid imaging in 1989, has become the radiopharmaceutical of choice for nuclear localization studies. Different techniques are used to differentiate sestamibi uptake by abnormal parathyroid glands from uptake by the thyroid gland. The double-phase technique relies on more rapid washout of sestamibi from thyroid tissue than from abnormal parathyroid glands. Images are obtained after the administration of sestamibi and then again approximately $2 \mathrm{~h}$ later (Fig. 1). The greater number of mitochondria in parathyroid tissue sequesters sestamibi intracellularly. High washout from the parathyroid tissue can result in false-negative findings. Subtraction imaging is performed with the coadministration of sestamibi and an agent for thyroid imaging, such as ${ }^{123} \mathrm{I}-$ or ${ }^{99 \mathrm{~m}} \mathrm{Tc}-$ pertechnetate. The thyroid images generated are then digitally subtracted from the sestamibi image. Residual signal after subtraction of the thyroid image is indicative of parathyroid uptake (8). Thyroid pathology, which can have increased sestamibi uptake and retention, may be mistaken as an abnormal parathyroid gland in the clinical setting of hyperparathyroidism. Correlation with anatomic imaging and the use of subtraction techniques are important in these cases.

Regardless of which technique is used, sestamibi scanning as a single modality for identifying adenomas has a reported sensitivity of $54 \%-100 \%$, with most series in the $80 \%$ or $90 \%$ range. Ultrasound alone as a single modality for the identification of adenomas has a reported sensitivity of $27 \%-89 \%$. Several series report a combined sensitivity with a range of $78 \%-96 \%(3,9,10)$. When localizing studies are undertaken,

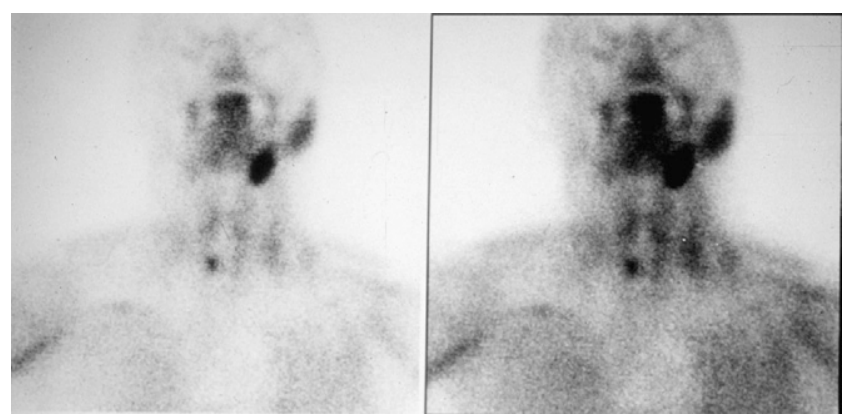

FIGURE 1. Image after administration of sestamibi (2-h delay on left window) demonstrates right inferior parathyroid adenoma. frequently a sestamibi scan is used in conjunction with some type of anatomic imaging, either ultrasound, CT, or MRI (Fig. 2). Among these anatomic imaging modalities, ultrasound has the advantage of not using ionizing radiation, having the lowest cost, and being the most frequently used imaging modality in this setting. It is, however, highly user-dependant and does not enable retromanubrial or mediastinal visualization. Invasive localizing studies, such as angiography and selective venous PTH assay, are rarely used now because of the high success rate of noninvasive approaches. Recently, several studies have reported the use of SPECT/CT for localization in patients with previously untreated primary hyperparathyroidism. The results have been variable, with some series demonstrating improved sensitivity and positive predictive value resulting in a change in therapeutic management and others reporting limited clinical value when evaluating patients with previously untreated primary hyperparathyroidism (11).

\section{TREATMENT}

The main treatment of primary hyperparathyroidism is surgery. The change in clinical presentation of primary hyperparathyroidism, from patients with grossly symptomatic disease to those who are largely asymptomatic but found incidentally to have abnormally elevated calcium and PTH levels, has spurred investigation into the natural history of the disease and the indications for treatment. It is generally agreed that surgery is indicated in patients who present with symptomatic disease such as nephrolithiasis, nephrocalcinosis, renal dysfunction, osteopenia with fractures, osteitis fibrosa cystica, and altered neurologic function with obtundation, delirium, or coma (2).

The management of patients with asymptomatic hyperparathyroidism has been controversial. To guide clinicians facing such patients, the National Institutes of Health organized a consensus development conference in 1990 to make recommendations on the treatment of primary hyperparathyroidism (12). In 2002, the National Institutes of Health convened another group, the Workshop on Asymptomatic Primary Hyperparathyroidism, to revisit these is-

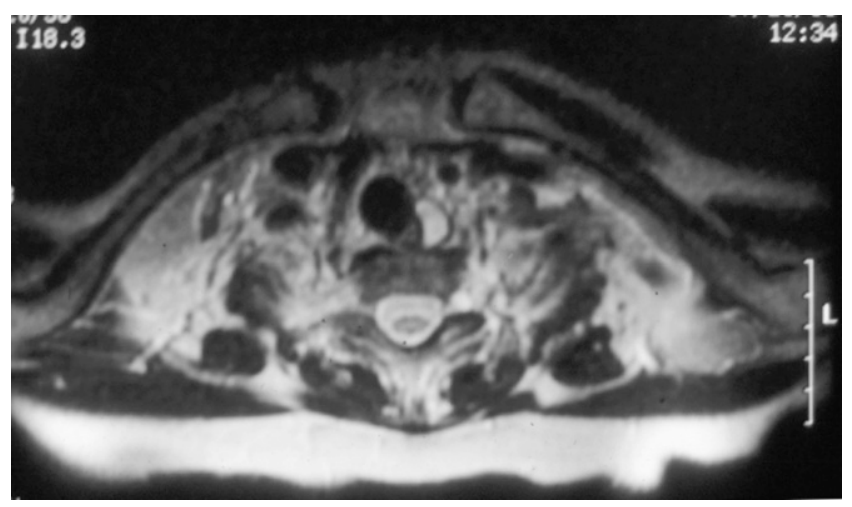

FIGURE 2. T2-weighted MR image demonstrates parathyroid adenoma in left tracheoesophageal groove. 
sues and make new recommendations. Revised consensus guidelines outlining the criteria for surgical treatment and management guidelines for observing patients with asymptomatic hyperparathyroidism (Table 2) were published (13, 14). Under these guidelines, the indications for surgery include a serum calcium concentration of $1.0 \mathrm{mg} / \mathrm{dL}$ above the upper limit of normal, a 24-h urinary calcium excretion of $400 \mathrm{mg}$ or more, a reduction in the creatinine clearance of more than $30 \%$, a bone mineral density with a $t$ score below -2.5 at any site, or age younger than $50 \mathrm{y}$. Patients with primary hyperparathyroidism should undergo dualenergy x-ray absorptiometry measurement of bone mineral density because a $t$ score less than 2.5 is an indication for surgical treatment. Patients found to have mild asymptomatic hyperparathyroidism and who do not fit any of the consensus criteria for surgical treatment can be observed with careful follow-up, which includes serum calcium evaluation every 6 mo and annual creatinine and dual-energy $\mathrm{x}$-ray absorptiometry testing.

Before the widespread use of sestamibi imaging and intraoperative rapid PTH assays, the treatment of primary hyperparathyroidism was bilateral neck exploration with the goal of locating and visually evaluating 4 parathyroid glands. Bilateral neck exploration generally involves making a transverse neck incision of 6-8 cm several centimeters above the clavicles, an incision similar to the one used for thyroid surgery. Both lobes of the thyroid gland are mobilized, both recurrent laryngeal nerves are identified, and the neck is explored to identify 4 parathyroid glands. The average weight of a parathyroid gland is approximately $35 \mathrm{mg}$, and the average diameter is between 1 and $5 \mathrm{~mm}$. Direct visualization is often sufficient for the identification of the abnormal gland or glands. Frozen section sampling can also be helpful for comparing an abnormal gland with a normal parathyroid. The 4-gland exploration approach has a high success rate, on the order of $95 \%(3,6)$.

\section{Minimally Invasive and Focused Surgical Approaches}

Because $85 \%$ or more of patients with primary hyperparathyroidism have a parathyroid adenoma, only 1 gland is

TABLE 2

Criteria for Parathyroid Surgery in Patients with Asymptomatic Primary Hyperparathyroidism

\begin{tabular}{|c|c|}
\hline Variable & Criterion \\
\hline $\begin{array}{l}\text { Serum calcium } \\
\text { concentration }\end{array}$ & $\begin{array}{l}1.0 \mathrm{mg} / \mathrm{dL} \text { above upper limit } \\
\text { of normal }\end{array}$ \\
\hline $\begin{array}{l}\text { 24-h urinary calcium } \\
\text { excretion }\end{array}$ & $>400 \mathrm{mg}$ \\
\hline $\begin{array}{l}\text { Reduction in creatinine } \\
\text { clearance }\end{array}$ & $>30 \%$ \\
\hline Bone mineral density & $t$ score below -2.5 at any site \\
\hline Age & $<50$ y \\
\hline \multicolumn{2}{|c|}{ Adapted from Bilezikian and Silverberg (2). } \\
\hline
\end{tabular}

responsible for their disease. If a sestamibi scan, in combination with anatomic imaging such as an ultrasound, indicates a single adenoma, then it is possible to focus the surgical attention in the area of the diseased gland (Fig. 3). A unilateral neck exploration involves making a smaller incision, generally $3-5 \mathrm{~cm}$, and exploring 1 side of the neck to identify both parathyroid glands and the recurrent laryngeal nerve. A focused single-gland exploration involves making an incision $2-4 \mathrm{~cm}$ long. Some surgeons do not search for the recurrent laryngeal nerve but rather look immediately for the single gland. With both unilateral neck exploration and single-gland exploration, rapid intraoperative measurement of PTH levels is used to increase the certainty that the gland or glands responsible for the patient's hyperparathyroidism are addressed at the time of surgery. The half-life of PTH in the circulation is approximately 2 min. Generally, a drop in PTH to less than 50\% of the measurement taken in the operating room before removal of the suspected gland indicates that the source of the hyperparathyroidism is removed. The blood draw after removal of the suspected adenoma should occur $10 \mathrm{~min}$ after the specimen is excised. An analysis of 210 published series using intraoperative PTH calculated that a unilateral neck dissection was completed as intended in $94.5 \%$ of the cases. Intraoperative PTH levels resulted in conversion to a bilateral neck exploration in $5.5 \%$ of cases. Persistent hypercalcemia after all cases in which intraoperative PTH was used was $1.3 \%(15)$.

Patients with 4-gland hyperplasia still require a bilateral neck exploration for a successful outcome (16). Either 3.5 parathyroid glands are excised, or all 4 glands are excised

Confirm diagnosis and complete evaluation

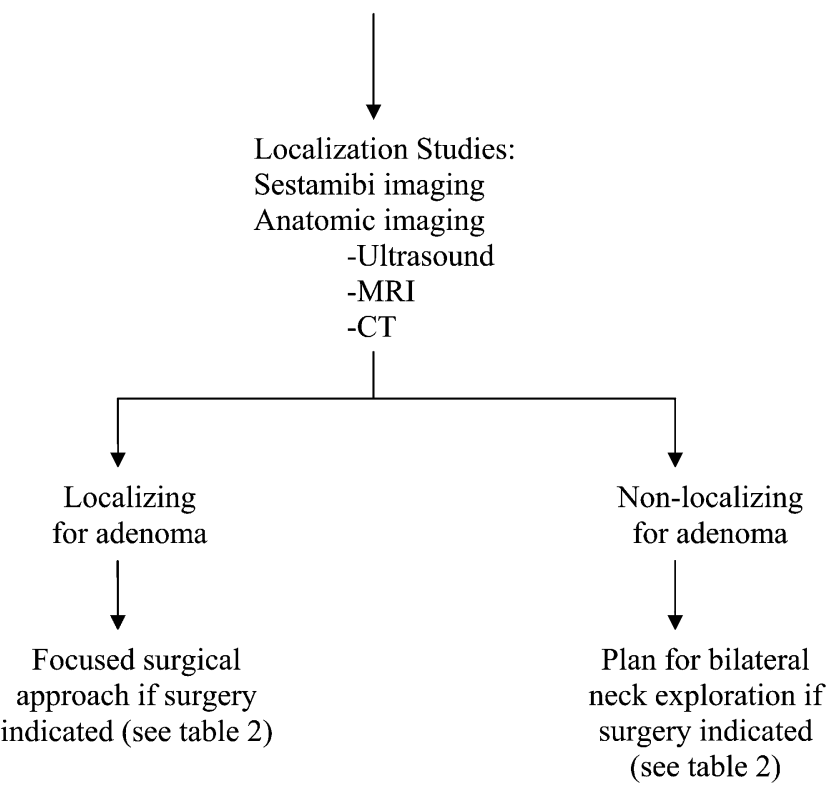

FIGURE 3. Management schema for primary hyperparathyroidism. 
with some parathyroid tissue reimplanted either in the neck, generally in the sternocleidomastoid muscle, or in the forearm. Any time a minimally invasive surgical approach is used, there is always a small chance that a bilateral neck exploration may be required to achieve a cure. For example, if imaging suggests a single adenoma, and a single-gland exploration is undertaken but the PTH levels do not drop adequately, there may be a second adenoma or the patient's underlying pathology may be hyperplasia of all 4 glands. In the case of 2 adenomas, removal of the second adenoma is necessary to achieve a cure.

\section{Endoscopic and Video-Assisted Parathyroid Surgery}

In the trend toward increasingly minimally invasive techniques, endoscopic parathyroidectomy has been developed at centers in which endoscopic techniques for thyroid surgery were practiced. Endoscopic techniques using gas insufflation have been reported, although these techniques are not common in the United States. Insufflation endoscopy allows incisions to be placed farther from the gland, such as below the clavicles or even in the axilla, in an effort to improve cosmesis (17). The disadvantage is that subcutaneous crepitus and dissection of gas into surrounding tissues can occur. A more frequently used endoscopic technique involves making a $1.5-$ to $2-\mathrm{cm}$ lateral cervical incision and placing an endoscopic instrument through this incision. Insufflation is not used, but an endoscopic assistant is required and the instruments are used to retract and dissect the tissues. The endoscopes provide greater magnification of the critical tissues for the surgeon, although they also require the surgeon to learn a new set of operative techniques. Rapid intraoperative PTH levels are measured to confirm that the overproducing gland has been removed, similar to other focused surgical techniques. A patient is generally considered a candidate if he or she has localizing studies strongly suggestive of a single parathyroid adenoma and anatomy that makes this approach difficult, such as obesity, thyroid enlargement or abnormality, or a short neck (18). Some authors refer to this endoscopic technique as minimally invasive videoassisted parathyroidectomy. A variation on this approach using a centrally placed incision that allows bilateral neck exploration has also been reported (19). Endoscopic and video-assisted parathyroidectomies are primarily performed to avoid a cervical incision. In many patients, especially those with skin creases in the neck, the cosmetic difference between a small-incision single-gland exploration and endoscopic approaches is probably minimal. Although not the standard of care, there are more centers around the country beginning to practice endoscopic or video-assisted parathyroidectomy.

\section{Radioguided Surgery}

Innovative techniques for radioguided surgery have been described and also continue to be used at some centers (20). Patients are given ${ }^{99 \mathrm{~m}}$ Tc-sestamibi, and imaging is performed on the day before surgery. A mark is made on the skin by the nuclear medicine physician, communicating to the surgeon the evidence of an adenoma. Intraoperatively, a $\gamma$-probe is used in the surgical wound to help guide the dissection (21). Usually a small 2- to $3-\mathrm{cm}$ incision is used in this single-gland exploration technique. When a suspected adenoma is removed, if the radioactivity is more than $20 \%$ of the background, it is considered by some surgeons to indicate successful adenoma removal (20).

\section{Anesthesia}

The traditional approach was to perform surgery with patients under general anesthesia; most parathyroidectomies, including those using focused surgical techniques, are still performed under general anesthesia using either endotracheal tube anesthesia or laryngeal mask anesthesia (18). Some centers, however, perform focused surgical techniques for parathyroid disease using monitored anesthesia care (MAC) sedation in combination with local and regional anesthetic blocks (22). Bilateral neck exploration in combination with thyroid procedures under MAC with local and regional blocks has also been described but is not commonly practiced (23). The low morbidity of general anesthesia is likely a principal reason that this approach continues as the most common practice. At some centers that use both general anesthesia and MAC with local and region blocks, patients are discharged on the same day as surgery, although more commonly patients are observed overnight (24).

\section{RECURRENT HYPERPARATHYROIDISM}

Regardless of the surgical approach taken to the treatment of hyperparathyroidism, no treatment is $100 \%$ curative. Patients with persistent or recurrent hyperparathyroidism previously operated on are difficult to treat successfully and-even more than routine patients with hyperparathyroidism-need a coordinated team for successful treatment (Fig. 4). Causes of a failed initial operation include incorrect diagnosis, an inexperienced surgeon, an undetected enlarged gland, multiple-gland disease, failure to detect an ectopic gland, supernumerary glands, parathyroid carcinoma, and parathymatosis from gland spillage during the initial procedure (25). The best strategy is to prevent the need for reoperative surgery in the first

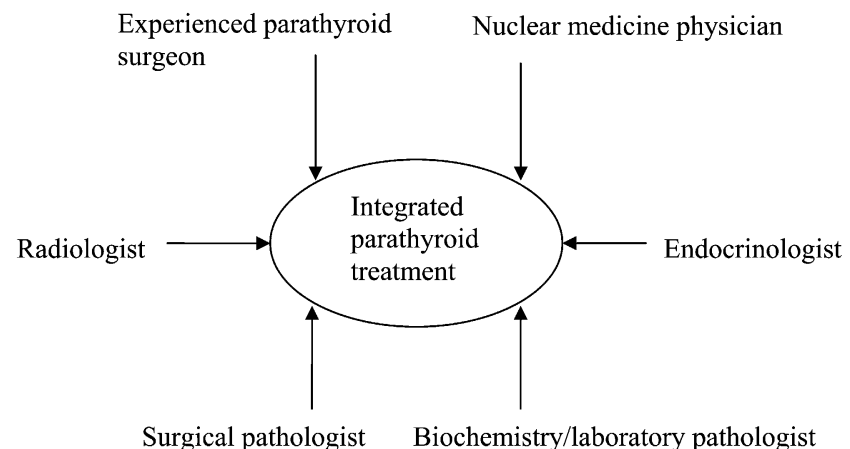

FIGURE 4. Multidisciplinary team approach in treatment of hyperparathyroidism. (Adapted from Shaha (6).) 
place. If, however, reoperation is required, the initial evaluation should be reviewed and a complete reevaluation should be performed, including sestamibi scanning and anatomic imaging, with consideration given to the possible causes of a failed initial operation. Particular attention should be paid to imaging the mediastinum with both sestamibi scanning and anatomic imaging. To provide successful care for patients with primary hyperparathyroidism and especially those for whom 1 surgical treatment has failed, it is necessary to have a team of physicians who understand this disease and work together, including an endocrinologist, a parathyroid surgeon, a nuclear medicine physician, anatomic radiologists, and clinical/laboratory pathologists (Fig. 4) (6).

\section{PARATHYROID CARCINOMA}

Parathyroid carcinoma is rare, and only a few reports on the treatment and outcomes of a small number of patients have been published. Preoperatively, the main clinical finding is high calcium and PTH levels. Nuclear imaging would likely localize a parathyroid carcinoma but has not been reported to have diagnostic utility. More frequently, these patients have symptomatic disease and can present in hypercalcemic crisis. The presence of metastatic disease to regional lymph nodes or distant sites can confirm the diagnosis before surgery on the primary tumor. The diagnosis can also be made intraoperatively on the basis of tumor invasion into surrounding structures. Histopathologic findings include a high mitotic rate and capsular, vascular, or neural invasion. Other findings such as cellular pleomorphism and atypia can also be seen. However, intraoperative examination of frozen sections remains controversial because of the difficulty of making the diagnosis with that technique.

\section{CONCLUSION}

The treatment of parathyroid disease is primarily surgical, but successful treatment must occur in the context of a team, including a radiologist skilled at performing sestamibi scanning and anatomic radiologists able to perform ultrasonography and other imaging modalities. The ability to perform rapid intraoperative PTH testing is also important. Endocrinologists are the experts in evaluating and diagnosing primary hyperparathyroidism. An experienced surgeon is also a critical member of the team.

\section{REFERENCES}

1. Melton GB, Somervell H, Friedman KP, Zeiger MA, Cahid Civelek A. Interpretation of ${ }^{99 \mathrm{~m} T c}$ sestamibi parathyroid SPECT scan is improved when read by the surgeon and nuclear medicine physician together. Nucl Med Commun. 2005;26:633-638.

2. Bilezikian JP, Silverberg SJ. Asymptomatic primary hyperparathyroidism. N Engl J Med. 2004;350:1746-1751.

3. McHenry CR. What's new in general surgery: endocrine surgery. $J$ Am Coll Surg. 2002;195:364-371.

4. Pasieka JL. What's new in general surgery: endocrine surgery. J Am Coll Surg. 2004;199:437-445.

5. Sosa JA, Zeiger MA. Surgery for hyperparathyroidism. Trends Endocrinol Metab. 1999;10:72-75.

6. Shaha AR. Parathyroid re-exploration. Otolaryngol Clin North Am. 2004; 37:833-843.

7. Silverberg SJ, Bilezikian JP. The diagnosis and management of asymptomatic primary hyperparathyroidism. Nat Clin Pract Endocirnol Metab. 2006;2:494-503.

8. Buck AK, Nekolla S, Ziegler S, et al. SPECT/CT. J Nucl Med. 2008;49:13051319.

9. Friedman K, Somervell H, Patel P, et al. Effect of calcium channel blockers on the sensitivity of preoperative ${ }^{99 \mathrm{~m}} \mathrm{Tc}-\mathrm{MIBI}$ SPECT for hyperparathyroidism. Surgery. 2004;136:1199-1204.

10. McHenry CR, Lee K, Saadey J, Neumann DR, Esselstyn CB Jr. Parathyroid localization with technetium-99m-sestamibi: a prospective evaluation. J Am Coll Surg. 1996;183:25-30.

11. Palestro CJ, Tomas MB, Tronco GG. Radionuclide imaging of the parathyroid glands. Semin Nucl Med. 2005;35:266-276.

12. AACE/AAES Task Force on Primary Hyperparathyroidism. The American Association of Clinical Endocrinologists and the American Association of Endocrine Surgeons position statement on the diagnosis and management of primary hyperparathyroidism. Endocr Pract. 2005;11:49-54.

13. Bilezikian JP, Potts JT Jr, Fuleihan Gel-H, et al. Summary statement from a workshop on asymptomatic primary hyperparathyroidism: a perspective for the 21st century. J Clin Endocrinol Metab. 2002;87:5353-5361.

14. Mahadevia PJ, Sosa JA, Levine MA, Zeiger MA, Powe NR. Clinical management of primary hyperparathyroidism and thresholds for surgical referral: a national study examining concordance between practice patterns and consensus panel recommendations. Endocr Pract. 2003;9:494-503.

15. Ruda JM, Hollenbeak CS, Stack BC. A systematic review of the diagnosis and treatment of primary hyperparathyroidism from 1995 to 2003. Otolaryngol Head Neck Surg. 2005;132:359-372.

16. Carling T, Udelsman R. Focused approach to parathyroidectomy. World J Surg. 2008;32:1512-1517.

17. Ikeda Y, Takami H, Niimi M, Kan S, Sasaki Y, Takayama J. Endoscopic thyroidectomy and parathyroidectomy by the axillary approach: a preliminary report. Surg Endosc. 2002;16:92-95.

18. Terris DJ, Stack BC Jr, Gourin CG. Contemporary parathyroidectomy: exploiting technology. Am J Otolaryngol. 2007;28:408-414.

19. Miccoli P, Berti P, Materazzi G, Ambrosini CE, Fregoli L, Donatini G. Endoscopic bilateral neck exploration versus quick intraoperative parathormone assay (qPTHa) during endoscopic parathyroidectomy: a prospective randomized trial. Surg Endosc. 2008;22:398-400.

20. Murphy C, Norman J. The $20 \%$ rule: a simple, instantaneous radioactivity measurement defines cure and allows elimination of frozen sections and hormone assays during parathyroidectomy. Surgery. 1999;126:1023-1028.

21. Chen H. Surgery for primary hyperparathyroidism: what is the best approach? Ann Surg. 2002;236:552-553.

22. Carling T, Donovan P, Rinder C, Udelsman R. Minimally invasive parathyroidectomy using cervical block: reasons for conversion to general anesthesia. Arch Surg. 2006;141:401-404.

23. Lo Gerfo P. Bilateral neck exploration for parathyroidectomy under local anesthesia: a viable technique for patients with coexisting thyroid disease with or without sestamibi scanning. Surgery. 1999;126:1011-1014.

24. Norman JG, Politz DE. Safety of immediate discharge after parathyroidectomy: a prospective study of 3,000 consecutive patients. Endocr Pract. 2007;13: $105-113$.

25. Carty SE. Prevention and management of complications in parathyroid surgery. Otolaryngol Clin North Am. 2004;37:897-907. 